\title{
Risk Determinants in Individuals With a Spontaneous Type 1 Brugada ECG
}

\author{
Akashi Miyamoto, MD; Hideki Hayashi, MD, PhD; Takeru Makiyama, MD, PhD; \\ Tomohide Yoshino, MD; Yuka Mizusawa, MD; Yoshihisa Sugimoto, MD, PhD; \\ Makoto Ito, MD, PhD; Joel Q Xue, PhD; Yoshitaka Murakami, PhD; Minoru Horie, MD, PhD
}

\begin{abstract}
Background: Spontaneous coved ST-segment elevation $\geq 2 \mathrm{~mm}$ followed by a negative T-wave in the right precordial leads (type 1 Brugada ECG) is diagnostic of Brugada syndrome (BS), but there is a false-positive rate.

Methods and Results: Computer-processed analysis of a 12-lead ECG database containing 49,286 females and 52,779 males was performed to select patients with a spontaneous type 1 Brugada ECG for an examination of the association of this ECG characteristic with long-term prognosis. There were 185 patients with a spontaneous type 1 Brugada ECG and of these, 16 (15 males; mean age, 46.7 14 .0 years) were diagnosed with BS and 15 patients (all males; mean age, $50.1 \pm 13.4$ years) were undiagnosed. The $P Q$ interval was significantly longer in the diagnosed patients than in the undiagnosed patients $(187.4 \pm 28.3 \mathrm{~ms}$ vs. $161.2 \pm 21.5 \mathrm{~ms} ; \mathrm{P}=0.0073)$. The $\mathrm{T}$-wave in lead $\mathrm{V}_{1}$ was more negative in the diagnosed patients than in the undiagnosed patients $(-170.2 \pm 174.6 \mu \mathrm{V}$ vs. $-43.2 \pm 122.3 \mu \mathrm{V}, \mathrm{P}=0.027$ ). Multivariate analysis revealed that a $\mathrm{PQ}$ interval $\geq 170 \mathrm{~ms}$ and $\mathrm{T}$-wave amplitude $<-105 \mu \mathrm{V}$ in lead $\mathrm{V}_{1}$ were independent risk stratifiers of life-threatening events. Survival analysis (mean follow-up, $78.6 \pm 81.8$ months) showed that the $P Q$ interval and a negative $\mathrm{T}$-wave in lead $\mathrm{V}_{1}$ were significantly associated with poor prognosis.
\end{abstract}

Conclusions: Analysis of a standard 12-lead ECG can stratify the prognosis of patients with a spontaneous type 1 Brugada ECG. (Circ J 2011; 75: 844-851)

Key Words: Brugada syndrome; Electrocardiography; Prognosis; Risk determinant; Sudden death

B rugada syndrome (BS) is characterized by a distinct ST-segment elevation in the right precordial leads and causes sudden cardiac death. ${ }^{1}$ This syndrome has a relatively high prevalence in East Asian countries. Patients with a coved-type ST-segment elevation in leads $\mathrm{V}_{1-3}$ are more susceptible to life-threatening ventricular arrhythmias than patients with a saddleback-type ST-segment elevation in the same leads. ${ }^{2}$ A community-based study reported that subjects who displayed a spontaneous coved ST-segment elevation in the right precordial leads were not at risk for sudden death, ${ }^{3}$ but another study, in which the mean followup period was $>40$ years, reported that an ECG with a coved ST-segment elevation was related to an increased risk of unexplained death. ${ }^{4}$ Similar inconsistency has been found among studies conducted in hospital-based populations. ${ }^{5-7}$ The discrepancy indicates that a distinct coved ST-segment elevation may not be the sole determinant of prognosis.

\section{Editorial $p 777$}

To stratify prognostic risk in BS, pharmacological and electrophysiological tests are performed, but the prognostic value of these tests is yet to be settled. ${ }^{8,9}$ In addition, mutation of the gene that encodes the cardiac sodium channel, $S C N 5 A,{ }^{10}$ is detected only in approximately $20 \%$ of patients diagnosed with $\mathrm{BS},{ }^{6}$ suggesting that it may be difficult to screen out subjects who are at high risk for lethal arrhythmia by genetic testing alone.

The large database of a university hospital containing 12lead ECGs of more than 100,000 patients stored digitally for over 25 years, enabled us to evaluate long-term prognosis using computer-processed analysis. Since 12-lead ECG is the most convenient method of diagnosing BS in a large population, such as in health examinations, in the present

Received September 8, 2010; revised manuscript received November 16, 2010; accepted December 1, 2010; released online February 18, 2011 Time for primary review: 16 days

Department of Cardiovascular and Respiratory Medicine, Shiga University of Medical Science, Otsu (A.M., H.H., T.Y., Y. Mizusawa, Y.S., M.I., M.H.); Department of Cardiovascular Medicine, Kyoto University Graduate School of Medicine, Kyoto (T.M.), Japan; General Electric Healthcare, Milwaukee, WI (J.Q.X.), USA; and Department of Health Science, Shiga University of Medical Science, Otsu (Y. Murakami), Japan

Mailing address: Hideki Hayashi, MD, PhD, Department of Cardiovascular and Respiratory Medicine, Shiga University of Medical Science, Otsu 520-2192, Japan. E-mail: hayashih@belle.shiga-med.ac.jp

ISSN-1346-9843 doi:10.1253/circj.CJ-10-0903

All rights are reserved to the Japanese Circulation Society. For permissions, please e-mail: cj@j-circ.or.jp 
study we focused on patients with a spontaneous coved STsegment elevation $\geq 0.2 \mathrm{mV}$ in the right precordial leads (ie, type 1 Brugada ECG). Our aim was to determine the quantitative traits of a spontaneous type 1 Brugada ECG that can stratify the risk for sudden cardiac death (SCD).

\section{Methods}

\section{Database}

The database comprised 102,065 consecutive patients $(49,286$ females and 52,779 males) who had undergone ECG recording in the university hospital between January 1983 and October 2008. A total of 308,391 ECGs were collected during this period. The 12-lead ECG was recorded at rest for $10 \mathrm{~s}$ at a sweep speed of $25 \mathrm{~mm} / \mathrm{s}$, calibrated to $1 \mathrm{mV} / \mathrm{cm}$ in the standard leads. The data were digitally stored in a 12-bit server computer with a sampling interval of $2 \mathrm{~ms}$.

\section{Patient Population}

From the database, we chose patients who had a spontaneous coved ST-segment elevation in the right precordial leads. We enrolled patients who fulfilled the following ECG criteria: (1) J-point elevation $\geq 0.2 \mathrm{mV}$ amplitude in lead $\mathrm{V}_{1}$ or $\mathrm{V}_{2}$, (2) the amplitude at the middle of the ST-segment (STM: defined as the time point after an interval of $1 / 16$ of the average $R R$ interval from the $J$ point) in lead $V_{1}$ or $V_{2}$ was less than that at the J point in the same lead, (3) the amplitude at the end of the ST-segment (defined as the time point after an interval of $1 / 8$ of the average RR interval from the $J$ point) in lead $V_{1}$ or $V_{2}$ was less than that at the middle of the ST-segment in the same lead, and (4) the amplitude at the J point and the middle of the ST-segment was positive in leads $\mathrm{V}_{1}$ and $V_{2}$. The $J$ point was defined as the offset of the QRS complex that was the latest detection of ventricular depolarization. To exclude right bundle branch block, we did not include any ECG that displayed the QRS complex in lead $\mathrm{V}_{1}$ with a decrease in amplitude of $\geq 0.4 \mathrm{mV}$ from the $\mathrm{J}$ point.

\section{ECG Analysis}

The ECG analysis was performed using software (MUSE7.1, GE Marquette Medical Systems, Inc, Milwaukee, WI, USA). ECG variables, including duration, interval, amplitude, and axis, were digitally measured. A median complex was computed as follows: (1) all QRS complexes with the same morphology were aligned in time and (2) the algorithm generated a representative QRS complex from the median voltages that were found at each successive sample time. QRS duration was measured from the earliest detection of depolarization in any lead (QRS onset) to the latest detection of depolarization in any lead (QRS offset). Similarly, the QT interval was measured from the earliest detection of depolarization in any lead (QRS onset) to the latest detection of repolarization where the downsloping limb joined the baseline in any lead (T offset), while the U wave was excluded. The QTc interval was calculated after correction for heart rate with Bazett's formula. The frontal plane angle of the P wave, QRS complex, and $\mathrm{T}$ wave was determined from the frontal leads ( $\mathrm{I}$, II, III, $\mathrm{aV}_{\mathrm{R}}, \mathrm{a} \mathrm{V}_{\mathrm{L}}$, and $\mathrm{aV}$ ). The frontal plane $\mathrm{QRS}-\mathrm{T}$ angle was defined as the absolute value of the difference between the frontal planes of the QRS axis and T axis, and was adjusted to the minimal angle using " $360^{\circ}$ minus the angle" for an angle $>180^{\circ}$ (axis measurement range; $-89^{\circ}$ to $+270^{\circ}$ ). Because all variables of the 12-lead ECG were digitally measured by computer-processed analysis, neither intra-observer nor interobserver variability was taken into account.

\section{Follow-up}

The follow-up period of all patients was defined as the interval from the first day when a spontaneous type 1 Brugada ECG was recorded to the day when prognostic outcome was identified. The prognostic value was assessed for the endpoint of documented ventricular fibrillation (VF) or sustained ventricular tachycardia (VT) that was either symptomatic or revealed by an implanted device. A postal questionnaire was used to assess the prognosis of patients who were not associated with the Division of Cardiology in the hospital. Written informed consent was given by all patients enrolled in this study.

\section{Diagnosis}

When a spontaneous type 1 Brugada ECG was present, BS was diagnosed on the basis of a consensus report requiring at least one of the following criteria: documented VF, self-terminating polymorphic VT, family history of sudden death, type 1 Brugada ECG in family members, positive electrophysiological study, unexplained syncope suggestive of ventricular tachyarrhythmia, and nocturnal agonal respiration. ${ }^{2}$ Confounding factors ${ }^{1}$ that have been previously reported as disorders accounting for a type 1 Brugada ECG were excluded. Drug-induced Brugada-like ECG pattern ${ }^{2}$ was also excluded.

\section{Gene Analysis}

The methods of DNA isolation and mutation analysis are described elsewhere. ${ }^{11}$ Briefly, genomic DNA was isolated from blood lymphocytes and then screened for candidate genes using denaturing high-performance liquid chromatography with a WAVE System Model 3500 (Transgenomic, Omaha, NB, USA). Polymerase chain reaction was used to amplify abnormal conformers, and sequencing was performed on an ABI PRISM 3100 DNA sequencer (Applied Biosystems, Foster City, CA, USA).

\section{Statistical Analysis}

We explored the prognostic factors for developing life-threatening events and the endpoint was the occurrence of lifethreatening events. Continuous variables are reported as mean \pm SD and categorical variables as observed number of patients (percentage). We compiled 2 groups: patients who were diagnosed with BS (diagnosis group) and patients who were not diagnosed with BS (no-diagnosis group). In the comparison of their clinical and ECG characteristics, we used a t-test for continuous variables and Fisher's exact test or $\chi^{2}$ test for categorical variables. Receiver-operating characteristic curve was used to determine the optimal cut-off point of the prognostic factors that maximizes the sensitivity and specificity of ECG variables for the diagnosis of BS. Logistic regression was used to compare the patients with/without BS to explore the prognostic factors accounting for confounding. The forward selection procedure was applied for the selection of variables in the logistic regression and the criteria was set as $\mathrm{P}<0.10$. For the significant variables in the model, the Kaplan-Meier curve was made to describe the event-free survival rate and the log-rank test was used to examine the difference between 2 groups. All tests were 2-tailed and the significance level was set as 0.5 . The research protocol was approved by the Ethical Committee of Shiga University of Medical Science (19-75).

\section{Results}

We located 185 patients who fulfilled the ECG criteria of a 


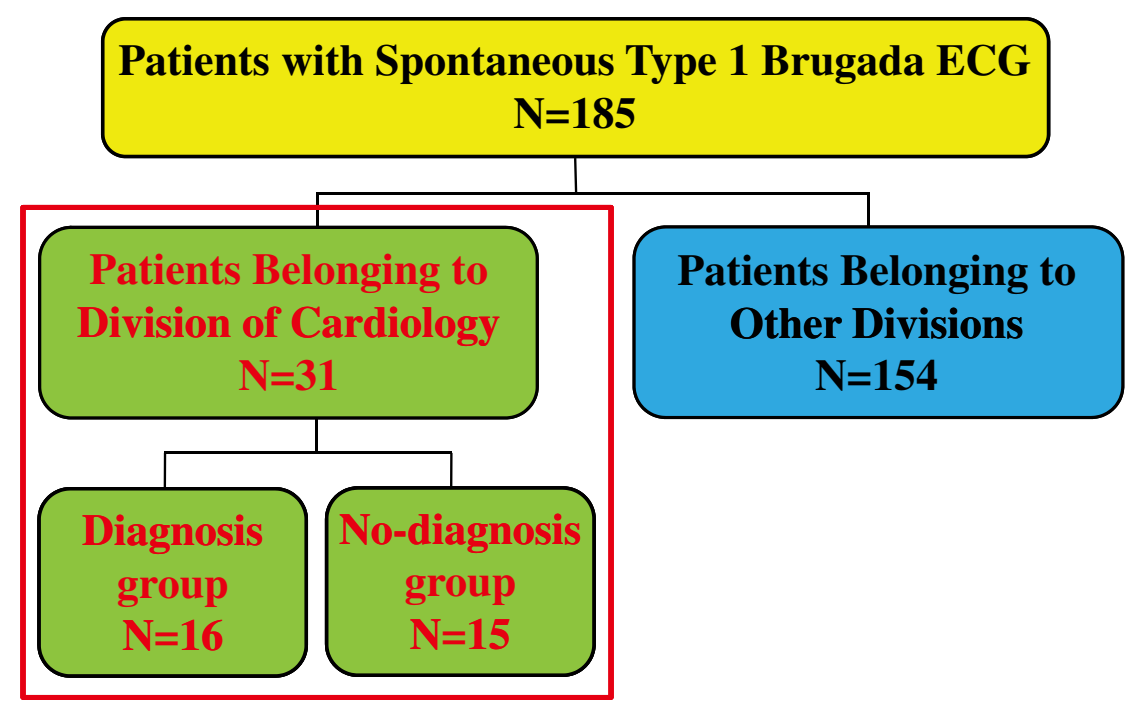

Figure 1. Flowchart of the categorization of patients with a spontaneous type 1 Brugada ECG: the 185 patients were chosen from an ECG database by virtue of a computer-processed algorithm. Of them, 31 patients were in the hospital's Division of Cardiology and 154 patients were not. The former patients were grouped: 16 patients who were diagnosed with Brugada syndrome (diagnosis group) and 15 patients who were not (no-diagnosis group). Red squares denote patients who were enrolled into this study.

\begin{tabular}{|lccc|}
\hline \multicolumn{2}{|l|}{ Table 1. Characteristics of the Patients With Spontaneous Type 1 Brugada ECG } & \\
No. of patients & Diagnosis group & No-diagnosis group & P value \\
Age (years) & 16 & 15 & \\
Male (n, \%) & $46.7 \pm 14.0$ & $50.1 \pm 13.4$ & 0.50 \\
Follow-up period (months) & $15(93.8)$ & $15(100)$ & 0.24 \\
Family history of SCD (n, \%) & $58.5 \pm 75.1$ & $43.2 \pm 42.7$ & 0.49 \\
Syncope (n, \%) & $8(50.0)$ & $0(0)$ & 0.0008 \\
Aborted SCD or documented VF (n, \%) & $14(87.5)$ & $3(20.0)$ & $<0.001$ \\
\hline
\end{tabular}

$\mathrm{SCD}$, sudden cardiac death; VF, ventricular fibrillation.

spontaneous type 1 Brugada ECG in the database (Figure 1). The prevalence of a spontaneous type 1 Brugada ECG was $0.18 \%$ of the total patients who underwent ECG recording in the hospital. Of the 185 patients, 31 attended the Division of Cardiology: 16 patients were diagnosed as BS (diagnosis group) and 15 patients were not (no-diagnosis group). Representative ECGs are shown in Figures S1 and S2. All the patients of the diagnosis and no-diagnosis groups were carefully followed in the Division of Cardiology. The remaining 154 patients who had exhibited a spontaneous type 1 Brugada ECG attended other divisions of the hospital and had undergone ECG recording irrespective of cardiovascular disease (eg, before surgery). The mean follow-up of the 2 groups was $51.1 \pm 61.1$ months, ranging from 1 to 238 months.

\section{Characteristics of the Patients}

The detailed clinical characteristics of each group are shown in Table 1. The mean age of each group was not significantly different, the male predominance was similar between the 2 groups and the mean follow-up period was not significantly different between the 2 cohorts. In the diagnosis group, 14 of the 16 patients diagnosed with BS suffered syncope, and in 12 of the 14 patients, lethal ventricular arrhythmias (VF or VT) were documented. Although the remaining 2 patients did not have a documented episode of lethal arrhythmias, both patients had syncopal episodes considered to be of arrhythmic origin. In those 2 patients, VF was induced by programmed ventricular stimulation. An implantable cardioverter-defibrillator (ICD) was implanted in all patients but 1 patient who rejected the procedure. After ICD implantation, 6 patients experienced VF. In the diagnosis group 8 patients $(50 \%$; 1 female, 7 males) had a family history of sudden death. In the no-diagnosis group, the 15 patients, who had been referred to the Division of Cardiology, displayed a spontaneous type 1 Brugada ECG but were not diagnosed as BS because none suffered from unexplained syncope or had a family history of SCD. Therefore, it was not considered necessary to implant ICDs in these patients, but they were required to visit hospital regularly for health checks. In the no-diagnosis group, 3 patients experienced syncope that was most likely due to a neurally mediated mechanism, because precipitating events, such as severe 


\begin{tabular}{lccl|}
\hline \multicolumn{4}{l}{ Table 2. Measurements of 12-Lead ECG of the Patients With Spontaneous Type 1 Brugada ECG } \\
Diagnosis group & No-diagnosis group & P value \\
Heart rate (beats/min) & $64.4 \pm 9.5$ & $68.3 \pm 14.7$ & 0.38 \\
PQ interval (ms) & $187.4 \pm 28.3$ & $161.2 \pm 21.5$ & 0.0073 \\
P axis (degrees) & $63.9 \pm 13.5$ & $58.7 \pm 19.5$ & 0.41 \\
QRS axis (degrees) & $49.1 \pm 44.1$ & $44.9 \pm 34.8$ & 0.78 \\
T axis (degrees) & $52.8 \pm 20.4$ & $59.3 \pm 14.0$ & 0.31 \\
QRS-T angle (degrees) & $19.8 \pm 17.3$ & $27.3 \pm 24.0$ & 0.32 \\
QRS-complex duration (ms) & $108.4 \pm 25.5$ & $103.2 \pm 8.6$ & 0.46 \\
QT interval (ms) & $398.1 \pm 34.9$ & $388.7 \pm 33.6$ & 0.45 \\
QTc interval (ms) & $409.3 \pm 28.2$ & $408.9 \pm 17.2$ & 0.96 \\
\hline
\end{tabular}

\begin{tabular}{|lccc|}
\hline \multicolumn{4}{|c|}{ Table 3. Measurements of Individual Leads of the Patients With Spontaneous Type 1 Brugada ECG } \\
QRS-complex duration in lead $\mathrm{V}_{1}(\mathrm{~ms})$ & Diagnosis group & No-diagnosis group & P value \\
QRS-complex duration in lead $\mathrm{V}_{2}(\mathrm{~ms})$ & $101.4 \pm 31.6$ & $90.3 \pm 19.1$ & 0.25 \\
QRS-complex duration in lead $\mathrm{V}_{5}(\mathrm{~ms})$ & $99.0 \pm 31.9$ & $88.9 \pm 18.1$ & 0.29 \\
Dispersion of QRS-complex duration $(\mathrm{ms})$ & $104.1 \pm 27.9$ & $98.3 \pm 11.4$ & 0.46 \\
R'-wave amplitude in lead $\mathrm{V}_{1}(\mu \mathrm{V})$ & $4.7 \pm 14.3$ & $7.9 \pm 21.9$ & 0.63 \\
R'-wave amplitude in lead $\mathrm{V}_{2}(\mu \mathrm{V})$ & $41.3 \pm 88.8$ & $94.9 \pm 138.4$ & 0.22 \\
J-point amplitude in lead $\mathrm{V}_{1}(\mu \mathrm{V})$ & $90.3 \pm 188.5$ & $188.9 \pm 248.9$ & 0.23 \\
J-point amplitude in lead $\mathrm{V}_{2}(\mu \mathrm{V})$ & $183.3 \pm 127.9$ & $128.8 \pm 78.0$ & 0.17 \\
STM amplitude in lead $\mathrm{V}_{1}(\mu \mathrm{V})$ & $311.8 \pm 117.6$ & $339.0 \pm 135.7$ & 0.55 \\
STM amplitude in lead $\mathrm{V}_{2}(\mu \mathrm{V})$ & $55.3 \pm 66.9$ & $81.7 \pm 73.9$ & 0.31 \\
Descending amplitude in lead $\mathrm{V}_{1}(\mu \mathrm{V})$ & $172.1 \pm 107.5$ & $192.9 \pm 157.8$ & 0.67 \\
T-wave amplitude in lead $\mathrm{V}_{1}(\mu \mathrm{V})$ & $128.0 \pm 154.0$ & $47.1 \pm 66.6$ & 0.071 \\
T-wave amplitude in lead $\mathrm{V}_{2}(\mu \mathrm{V})$ & $-170.2 \pm 174.6$ & $-43.2 \pm 122.3$ & 0.027 \\
R-wave amplitude in lead $\mathrm{a} \mathrm{V}_{\mathrm{R}}(\mu \mathrm{V})$ & $95.0 \pm 363.3$ & $232.1 \pm 200.0$ & 0.21 \\
R/q ratio in lead a $\mathrm{V}_{\mathrm{R}}$ & $104.5 \pm 142.8$ & $103.7 \pm 101.3$ & 0.99 \\
R-wave amplitude in lead $\mathrm{a} \mathrm{V}_{\mathrm{L}}(\mu \mathrm{V})$ & $0.35 \pm 0.44$ & $0.22 \pm 0.20$ & 0.38 \\
R'-wave amplitude in lead $\mathrm{V} \mathrm{V}_{L}(\mu \mathrm{V})$ & $254.3 \pm 281.0$ & $212.7 \pm 192.9$ & 0.64 \\
R'/q ratio in lead a $\mathrm{V}_{\mathrm{L}}$ & $32.2 \pm 48.4$ & $49.9 \pm 88.4$ & 0.49 \\
\hline
\end{tabular}

Dispersion of QRS-complex duration, absolute value of the difference between the QRS-complex duration in leads $\mathrm{V}_{1}$ and $\mathrm{V}_{5}$; STM, site at the middle of the ST segment (see Methods); Descending amplitude, difference in the amplitudes at the J-point and STM.

pain, emotional distress, or supine posture, were associated with syncope that was preceded by prodromal symptoms (sweating, nausea, vomiting, yawning). Cardiac transthoracic echocardiography revealed normal left ventricular function without evidence of structural heart disease in all patients of both groups. None of the family members of patients in either group was involved. We performed genetic analysis of 14 of the 16 patients in the diagnosis group, which identified a mutation in SCN5A in 1 patient $(8.3 \%)$ who was a subject of our previous report; ${ }^{12}$ no abnormality in $S C N 5 A$ was determined in the remaining 13 patients.

\section{Characteristics of the ECG}

Table 2 shows the ECG characteristics. Heart rate did not significantly differ between groups. The PQ interval was significantly longer in the diagnosis group than in the no-diagnosis group; however, there was no significant difference between the 2 groups in the duration of the QRS complex, QT interval, and QTc interval. In addition, the frontal plane axis of the P-, QRS-, and T-wave did not differ between the diagnosis and no-diagnosis group. Table 3 shows the ECG measurements in individual leads. Between the 2 groups, there was no significant difference in the duration of the QRS complex in leads $V_{1}, V_{2}$ and $V_{5}$, nor was there in the dispersion of the QRS-complex duration in leads $\mathrm{V}_{1}$ and $\mathrm{V}_{5}$, the $\mathrm{R}$ '-wave amplitude in leads $\mathrm{V}_{1}$ and $\mathrm{V}_{2}$, the J-point amplitude in leads $V_{1}$ and $V_{2}$, and the STM-amplitude in leads $V_{1}$ and $\mathrm{V}_{2}$. The $\mathrm{T}$-wave in lead $\mathrm{V}_{1}$ was more negative in the diagnosis group than in the no-diagnosis group, but the T-wave amplitude in lead $\mathrm{V}_{2}$ was not significantly different between the 2 groups. There was no significant difference between the 2 groups in the other ECG variables, including R-wave amplitude and $\mathrm{R} / \mathrm{q}$ ratio in lead $\mathrm{a} \mathrm{V}_{\mathrm{R}}$, and $\mathrm{R}$-wave amplitude, $\mathrm{R}$ '-wave amplitude, and R'/q ratio in lead $\mathrm{aV}$.

\section{Risk Stratification}

In the patients of the diagnosis and no-diagnosis groups, the factors predictive of life-threatening arrhythmic events were evaluated. Univariate analyses were performed to identify patients at risk of life-threatening events. The presence of a family history, and a PQ interval, and the T-wave amplitude in lead $\mathrm{V}_{1}$ were significantly associated with BS (Table 4). Multivariate logistic regression analysis revealed that the PQ interval and $\mathrm{T}$-wave amplitude in lead $\mathrm{V}_{1}$ were indepen- 


\begin{tabular}{|c|c|c|c|}
\hline & $P$ value & Odds ratio & $95 \% \mathrm{Cl}$ \\
\hline \multicolumn{4}{|l|}{ Univariate analysis } \\
\hline Age $\geq 50$ years & 0.36 & 1.93 & $0.47-8.42$ \\
\hline Family history of sudden death & $0.043^{*}$ & 10.50 & $1.47-216.68$ \\
\hline Heart rate $\geq 66$ beats $/ \mathrm{min}$ & 0.59 & 1.47 & $0.36-6.24$ \\
\hline$P Q$ interval $\geq 170 \mathrm{~ms}$ & $0.0014^{\star}$ & 14.0 & $2.62-115.18$ \\
\hline QTc interval $\geq 405 \mathrm{~ms}$ & 0.36 & 0.52 & $0.12-2.14$ \\
\hline J-point amplitude in $\mathrm{V}_{1} \geq 155 \mu \mathrm{V}$ & 0.85 & 0.86 & $0.15-3.22$ \\
\hline STM amplitude in lead $\mathrm{V}_{1} \geq 68 \mu \mathrm{V}$ & 0.58 & 0.67 & $0.16-2.76$ \\
\hline T-wave amplitude $<-105 \mu \mathrm{V}$ in lead $\mathrm{V}_{1}$ & $0.024^{*}$ & 6.05 & $1.36-32.09$ \\
\hline Descending amplitude in lead $\mathrm{V}_{1} \geq 49 \mu \mathrm{V}$ & 0.59 & 1.47 & $0.36-6.24$ \\
\hline \multicolumn{4}{|l|}{ Multivariate analysis } \\
\hline$P Q$ interval $\geq 170 \mathrm{~ms}$ & $0.045^{\star}$ & 11.50 & $1.05-268.62$ \\
\hline T-wave amplitude $<-105 \mu \mathrm{V}$ in lead $\mathrm{V}_{1}$ & $0.037^{\star}$ & 8.98 & $1.14-117.28$ \\
\hline Descending amplitude in lead $\mathrm{V}_{1} \geq 49 \mu \mathrm{V}$ & 0.19 & 0.23 & $0.02-1.94$ \\
\hline Family history of sudden death & 0.59 & 2.18 & $0.12-66.22$ \\
\hline
\end{tabular}

Dispersion of QRS-complex duration, absolute value of the difference between the QRS-complex duration in leads $\mathrm{V}_{1}$ and $V_{5}$; STM, site at the middle of the ST segment (see Methods); Descending amplitude, difference in the amplitudes at the J-point and STM.

$\mathrm{Cl}$, confidence interval.

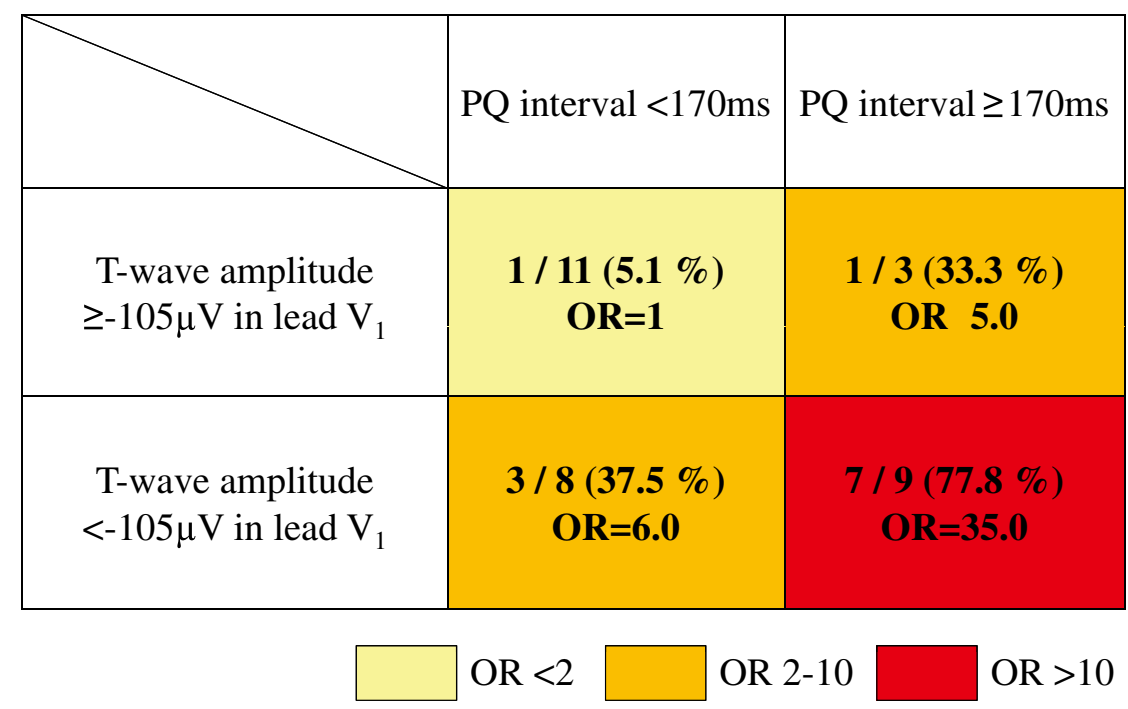

Figure 2. Risk stratification scheme of life-threatening events in patients with a spontaneous type 1 Brugada ECG. Patients are categorized according to the PQ interval and T-wave amplitude in lead $V_{1}$. Each box shows the number of patients of the diagnosis and no-diagnosis groups as a denominator and the number of patients of the diagnosis group as a numerator, allowing calculation of the rate of diagnosing Brugada syndrome (shown in parentheses). The odds ratio (OR) for each box is calculated by setting the odds of the upper lefthand corner yellow box as 1. See text for details.

dently associated with life-threatening events in patients with a spontaneous type 1 Brugada ECG (Table 4). Figure 2 is a schema for risk stratification constructed according to the values of the PQ interval and T-wave amplitude in lead $\mathrm{V}_{1}$. Using receiver operating characteristic analysis, the sensitivity and specificity of the PQ interval and T-wave amplitude in lead $\mathrm{V}_{1}$ in response to life-threatening events were maximized by a PQ interval of $170 \mathrm{~ms}$ and a T-wave amplitude of $-105 \mu \mathrm{V}$ in lead $\mathrm{V}_{1}$. The patients of the diagnosis and no- diagnosis groups were allocated to 4 categories according to the PQ interval and T-wave amplitude in lead $\mathrm{V}_{1}$. The rate of diagnosing BS was higher in the category having a PQ interval $\geq 170 \mathrm{~ms}$ than in that of PQ interval $<170 \mathrm{~ms}$. The same was true for the categories dichotomized by T-wave amplitude of $-105 \mu \mathrm{V}$ in lead $\mathrm{V}_{1}$. Furthermore, when present together, the PQ interval and T-wave amplitude in lead $\mathrm{V}_{1}$ potentiate each other, leading to a diagnosis of BS that is substantially greater than that of its individual components. 


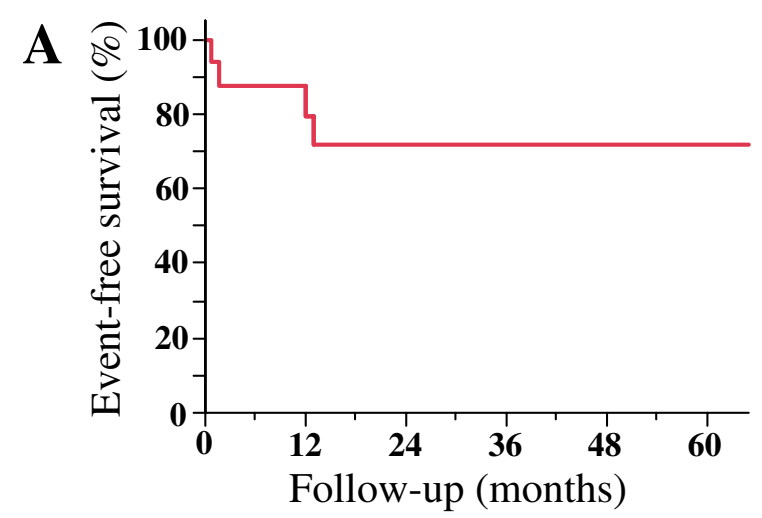

No. of patients

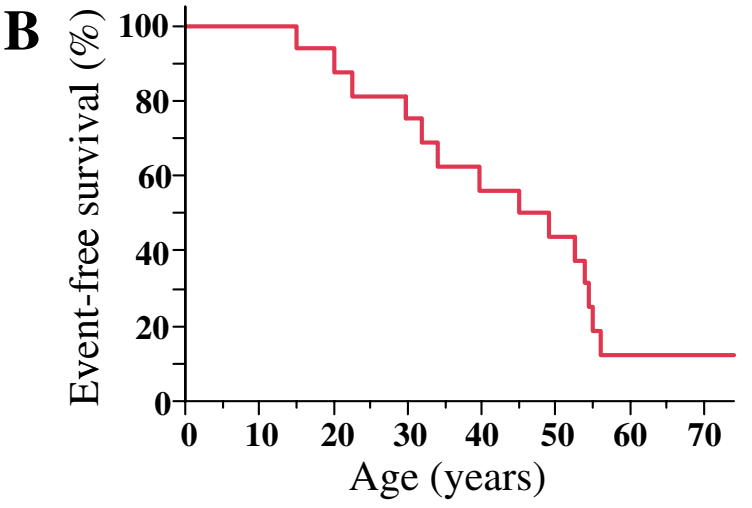

No. of patients

Figure 3. (A) Kaplan-Meier curve of event-free survival in patients with Brugada syndrome during follow-up. On the abscissa, time 0 is defined as the time when the first ECG with a spontaneous type 1 Brugada ECG was recorded. (B) Kaplan-Meier curve of event-free survival in patients with Brugada syndrome according to age.

\section{A}

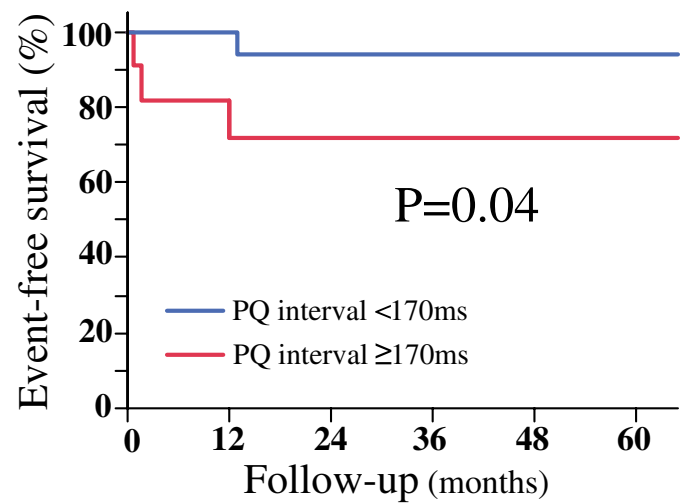

No. of patients

Follow-up (months)

$\begin{array}{rrrrrr}20 & 18 & 14 & 9 & 4 & 3 \\ 11 & 8 & 6 & 5 & 3 & 3\end{array}$

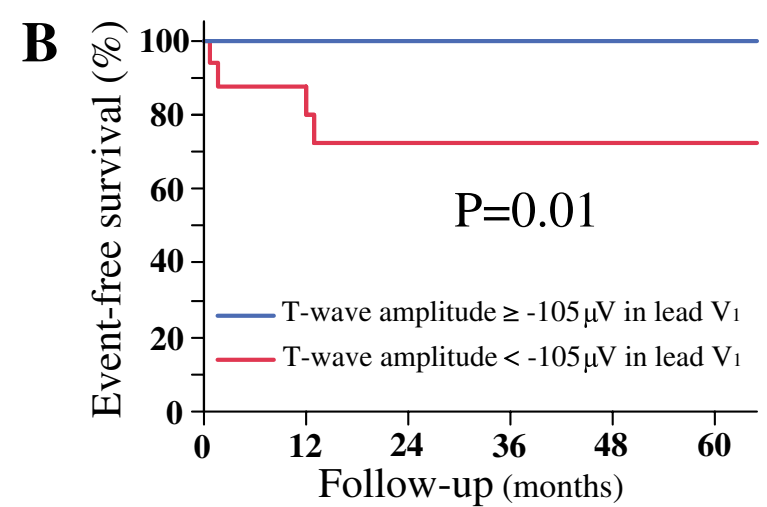

No. of patients

$\begin{array}{cccccc}15 & 14 & 12 & 10 & 5 & 4 \\ 16 & 12 & 8 & 4 & 2 & 2\end{array}$

Figure 4. Kaplan-Meier estimates of cardiovascular event-free survival in patients of the diagnosis and no-diagnosis groups during follow-up according to (A) PQ interval and (B) T-wave amplitude in lead $\mathrm{V}_{1}$.

\section{Long-Term Outcome}

Figure 3A shows the Kaplan-Meier survival curve of lifethreatening events in the diagnosis group after a type 1 Brugada ECG was recorded. During the follow-up, 6 of the 15 patients $(40 \%)$ who received an ICD experienced recurrence of life-threatening arrhythmic events. No patient died in the diagnosis group during follow-up. The duration from the diagnosis of BS to recurrence of life-threatening events

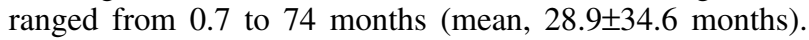
Approximately $30 \%$ of patients had recurrence within 1 year (Figure 3A). Figure 3B shows the age-dependent event-free survival curve of life-threatening events in the diagnosis group. Life-threatening events occurred between 15 and 60 years of age. A comparison of the ECG variables of patients with ICD intervention with those of patients without an ICD in the diagnosis group was made (Table S1). Heart rate was significantly faster in patients with an ICD than in those without, and the PQ interval was significantly longer in patients with an ICD than in those without. However, other ECG variables did not differ between patients with and without an ICD.

On the basis of the significant association of PQ interval and negative $\mathrm{T}$ wave in lead $\mathrm{V}_{1}$ with $\mathrm{BS}$, as shown in Table 4, the life-threatening event-free rate was estimated for the patients in both groups according to PQ interval and T-wave amplitude in lead V1. Figure 4 shows the KaplanMeier life-table analysis. The PQ interval was associated with a significant $(\mathrm{P}=0.04)$ difference in the life-threatening event-free rate between patients $(n=11)$ with a PQ interval $\geq 170 \mathrm{~ms}$ and those $(\mathrm{n}=20)$ with a PQ interval $<170 \mathrm{~ms}$ (haz- 
ard ratio, 6.9; 95\% CI, 1.1-133.5) (Figure 4A). In addition, the $\mathrm{T}$-wave amplitude in lead $\mathrm{V}_{1}$ was associated with a significant $(\mathrm{P}=0.013)$ difference in the life-threatening eventfree rate between patients $(n=16)$ with an amplitude $<-105 \mu \mathrm{V}$ and those $(\mathrm{n}=15)$ with an amplitude $\geq-105 \mu \mathrm{V}$ (hazard ratio, not available [because of the lack of events in patients with a $\mathrm{T}$-wave amplitude in lead $\left.\mathrm{V}_{1} \geq-105 \mu \mathrm{V}\right]$ ) (Figure 3B). Because none of the patients in the no-diagnosis group had a life-threatening event, multivariate survival analysis could not be performed.

\section{Discussion}

To utilize the convenience of the 12-lead ECG, a spontaneous type 1 Brugada ECG was used to enroll patients in this study. By conducting a computer-processed analysis, the discriminative ECG features of patients diagnosed with BS (diagnosis group), compared with patients not diagnosed with BS (no-diagnosis group), were found. First, atrial conduction was delayed in patients diagnosed with BS as compared with the no-diagnosis group. Second, the T-wave in lead $\mathrm{V}_{1}$ was more negative in patients diagnosed with $\mathrm{BS}$. Third, the duration of the QRS-complex in the right precordial leads did not show a significant difference between the diagnosed and undiagnosed patients. In addition, the amplitude of the R'-wave, J-point, and STM in the right precordial leads did not show a significant difference between the diagnosed and undiagnosed patients. These ECG findings are novel for differentiating patients at risk of developing lifethreatening arrhythmia among patients who show a spontaneous type 1 Brugada ECG. Moreover, the risk-stratification schemes elaborated in this study revealed the patients who needed an ICD.

\section{Risk Determinants}

In BS, the transient outward current ( $\mathrm{I}_{\mathrm{to}}$ )-mediated phase 1 is much more prominent in the epicardium than in the endocardium, leading to ST-segment elevation in the right precordial leads. ${ }^{11}$ However, our data from the present study showed there was no significant difference in either the J-point amplitude or the ST-segment amplitude in the right precordial leads between patients diagnosed or not diagnosed with BS. There are several reasons for this: (1) we enrolled patients with a J-point amplitude $\geq 2 \mathrm{~mm}$ and covedtype ST-segment elevation, (2) the ST-segment elevation could be due to ion channel abnormalities such as a reduction of sodium and calcium currents, and (3) a clinically veiled histological abnormality may affect ventricular repolarization. In contrast to the amplitude of the J-point and the $\mathrm{ST}$ segment, the $\mathrm{T}$-wave in lead $\mathrm{V}_{1}$ was more negative in patients diagnosed with BS than in the undiagnosed patients. This finding is compatible with the inward calcium current overcoming the outward Ito during phases 1 and 2, causing a secondary depolarization in the epicardial action potential. Besides, the balance of the 2 ionic currents reverses as the Ito current overwhelms the calcium current, resulting in a loss of the action potential dome and an abbreviation of the action potential duration that lead to phase 2 reentry..$^{13}$ Consistent with these fundamental mechanisms, Nagase et $\mathrm{al}^{14}$ demonstrated that prolongation of the epicardial action potential following Ito-mediated accentuation of the action potential in the right ventricular outflow tract caused the T-wave in the right precordial leads to become negative, coinciding with a type 1 Brugada ECG. In addition, dynamic instability depicted by the restitution property of the action potential duration in the right ventricular outflow tract may contribute to the occurrence of reentry. ${ }^{15}$

Though the patients diagnosed with BS did not show significant intraventricular conduction delay in the right ventricle, which was different from a previous report, ${ }^{16}$ the atrial conduction delay was more pronounced in the diagnosed patients than in the undiagnosed patients. It has been reported that there is an increased atrial vulnerability to fibrillation in BS. ${ }^{17,18}$ In those reports, the inter- and intra-atrial conduction delays were associated with atrial fibrillation. In the present study, the PQ interval was longer in the diagnosis group than in the no-diagnosis group, but the QRS-complex duration did not differ between the 2 groups. These findings suggest that a conduction disturbance occurred in the atrium and/or the atrioventricular node rather than in the ventricle. In fact, atrial fibrillation occurred only in 1 patient of each group during the follow-up. We should therefore pay close attention to examining whether atrial fibrillation develops. Furthermore, a P-wave abnormality ${ }^{19}$ and a high prevalence of sick sinus syndrome ${ }^{20}$ complicated by $\mathrm{BS}$ indicate atrial involvement.

\section{Long-Term Prognosis}

Brugada et al showed that symptomatic and asymptomatic patients with ST-segment elevation in the right precordial leads shared a similar incidence of cardiac arrest. ${ }^{21}$ Other investigators $\mathrm{s}^{6,7}$ also reported that asymptomatic patients with such an ECG characteristic were at risk for sudden death, although the event-free survival rate in those studies was much lower than that of patients in the "Brugada" registry. ${ }^{13}$ In contrast, sudden death did not occur in any of patients of the no-diagnosis group and asymptomatic group in our study. This result may be related to not involving family members of proband in the study.

Similar to previous reports, ${ }^{6,7}$ we found that most patients of the diagnosis group suffered from ventricular tachyarrhythmia or syncope of unknown origin and approximately one-third patients of the diagnosis group had a recurrence of ventricular tachyarrhythmia. In contrast, none of patients not diagnosed with BS (no-diagnosis group) had sudden death or ventricular tachyarrhythmia. Thus, we emphasize again the importance of medical history-taking: syncope and family history of sudden death.

\section{Study Limitations}

First, the ST-segment elevation is not constantly observed in BS patients, because of the so-called "wax and wane" phenomenon, therefore patients with an ST-segment elevation $<0.2 \mathrm{mV}$ at the J-point were missed even if they had BS. Second, because of the limited follow-up, it cannot be assumed that asymptomatic patients did not develop SCD. Third, the response bias of the questionnaire should be considered. We must pay further attention to assessing the longterm prognosis in asymptomatic patients with a spontaneous type 1 Brugada ECG.

\section{Study Implications}

Despite the fact that a spontaneous type 1 Brugada ECG is diagnostic, the discriminative ECG features associated with a risk for SCD remain undetermined. From the results of the present study, we propose the PQ interval and negative $\mathrm{T}$ wave in lead $V_{1}$ as valuable ECG markers of BS. In addition, we underscore that medical information, including the family history, is helpful in the management of patients with a spontaneous type 1 Brugada ECG. It may be possible to deduce 
the ECG features presented here to locate subjects in wide populations, such as health examinations, who are at risk.

\section{Acknowledgments}

The authors thank Kahaku Emoto, Seiichi Fujisaki, and Tatsumi Uchiyama (GE Yokokawa Medical System Co) for their technical assistance.

\section{Disclosures}

No conflicts to disclose.

\section{References}

1. Brugada P, Brugada J. Right bundle branch block, persistent ST segment elevation and sudden cardiac death: A distinct clinical and electrocardiographic syndrome: A multicenter report. J Am Coll Cardiol 1992; 20: 1391-1396.

2. Antzelevitch C, Brugada P, Borggrefe M, Brugada J, Brugada R, Corrado D, et al. Brugada syndrome: Report of the second consensus conference: Endorsed by the heart rhythm society and the european heart rhythm association. Circulation 2005; 111: 659-670.

3. Miyasaka Y, Tsuji H, Yamada K, Tokunaga S, Saito D, Imuro Y, et al. Prevalence and mortality of the Brugada-type electrocardiogram in one city in japan. J Am Coll Cardiol 2001; 38: 771-774.

4. Matsuo K, Akahoshi M, Nakashima E, Suyama A, Seto S, Hayano $\mathrm{M}$, et al. The prevalence, incidence and prognostic value of the Brugada-type electrocardiogram: A population-based study of four decades. J Am Coll Cardiol 2001; 38: 765-770.

5. Brugada J, Brugada R, Antzelevitch C, Towbin J, Nademanee K, Brugada P. Long-term follow-up of individuals with the electrocardiographic pattern of right bundle-branch block and ST-segment elevation in precordial leads V1 to V3. Circulation 2002; 105: $73-78$.

6. Priori SG, Napolitano C, Gasparini M, Pappone C, Della Bella P, Giordano U, et al. Natural history of Brugada syndrome: Insights for risk stratification and management. Circulation 2002; 105: $1342-$ 1347.

7. Eckardt L, Probst V, Smits JP, Bahr ES, Wolpert C, Schimpf R, et al. Long-term prognosis of individuals with right precordial STsegment-elevation brugada syndrome. Circulation 2005; 111: 257 263.

8. Brugada P, Brugada R, Brugada J. Should patients with an asymptomatic Brugada electrocardiogram undergo pharmacological and electrophysiological testing? Circulation 2005; 112: 279-292; discussion 279-292.

9. Priori SG, Napolitano C. Should patients with an asymptomatic Brugada electrocardiogram undergo pharmacological and electrophysiological testing? Circulation 2005; 112: 279-292; discussion 279-292.

10. Chen Q, Kirsch GE, Zhang D, Brugada R, Brugada J, Brugada P, et al. Genetic basis and molecular mechanism for idiopathic ventricular fibrillation. Nature 1998; 392: 293-296.

11. Makiyama T, Akao M, Shizuta S, Doi T, Nishiyama K, Oka Y, et al. A novel scn5a gain-of-function mutation M1875T associated with familial atrial fibrillation. J Am Coll Cardiol 2008; 52: 13261334.

12. Kawamura M, Ozawa T, Yao T, Ashihara T, Sugimoto Y, Yagi T, et al. Dynamic change in ST-segment and spontaneous occurrence of ventricular fibrillation in Brugada syndrome with a novel nonsense mutation in the SCN5A gene during long-term follow-up. Circ J 2009; 73: 584-588.

13. Yan GX, Antzelevitch C. Cellular basis for the Brugada syndrome and other mechanisms of arrhythmogenesis associated with STsegment elevation. Circulation 1999; 100: 1660-1666.

14. Nagase S, Kusano KF, Morita H, Nishii N, Banba K, Watanabe A, et al. Longer repolarization in the epicardium at the right ventricular outflow tract causes type 1 electrocardiogram in patients with Brugada syndrome. J Am Coll Cardiol 2008; 51: 1154-1161.

15. Ashino S, Watanabe I, Kofune M, Nagashima K, Ohkubo K, Okumura Y, et al. Abnormal action potential duration restitution property in the right ventricular outflow tract in Brugada syndrome. Circ J 2010; 74: 664-670.

16. Kanda M, Shimizu W, Matsuo K, Nagaya N, Taguchi A, Suyama K, et al. Electrophysiologic characteristics and implications of induced ventricular fibrillation in symptomatic patients with Brugada syndrome. J Am Coll Cardiol 2002; 39: 1799-1805.

17. Morita H, Kusano-Fukushima K, Nagase S, Fujimoto Y, Hisamatsu $\mathrm{K}$, Fujio $\mathrm{H}$, et al. Atrial fibrillation and atrial vulnerability in patients with Brugada syndrome. J Am Coll Cardiol 2002; 40: 1437-1444.

18. Kusano KF, Taniyama M, Nakamura K, Miura D, Banba K, Nagase $\mathrm{S}$, et al. Atrial fibrillation in patients with Brugada syndrome relationships of gene mutation, electrophysiology, and clinical backgrounds. J Am Coll Cardiol 2008; 51: 1169-1175.

19. Yamada T, Watanabe I, Okumura Y, Takagi Y, Okubo K, Hashimoto $\mathrm{K}$, et al. Atrial electrophysiological abnormality in patients with Brugada syndrome assessed by P-wave signal-averaged ECG and programmed atrial stimulation. Circ J 2006; 70: 1574-1579.

20. Hayashi H, Sumiyoshi M, Yasuda M, Komatsu K, Sekita G, Kawano Y, et al. Prevalence of the Brugada-type electrocardiogram and incidence of Brugada syndrome in patients with sick sinus syndrome. Circ J 2010; 74: 271-277.

21. Brugada J, Brugada R, Brugada P. Right bundle-branch block and ST-segment elevation in leads V1 through V3: A marker for sudden death in patients without demonstrable structural heart disease. Circulation 1998; 97: 457-460.

\section{Supplemental Files}

Supplemental File 1

Figure S1. 12-lead ECG of a 49-year-old man who was diagnosed with Brugada syndrome.

Figure S2. 12-lead ECG of a 55-year-old man who was undiagnosed with Brugada syndrome.

Table S1. Comparison of ECG Variables Between Patients With and Without ICD Intervention in the BS Diagnosis Group

Please find supplemental file(s);

http://dx.doi.org/10.1253/circj.CJ-10-0903 